LA W REN CE LIVERMORE N A T IO N A L LABORATORY

Thoughts on Uncertainties in the

Moments Formalism of the Statistical

Theory of Fission Chains

S. Walston

November 4, 2013 
This document was prepared as an account of work sponsored by an agency of the United States government. Neither the United States government nor Lawrence Livermore National Security, LLC, nor any of their employees makes any warranty, expressed or implied, or assumes any legal liability or responsibility for the accuracy, completeness, or usefulness of any information, apparatus, product, or process disclosed, or represents that its use would not infringe privately owned rights. Reference herein to any specific commercial product, process, or service by trade name, trademark, manufacturer, or otherwise does not necessarily constitute or imply its endorsement, recommendation, or favoring by the United States government or Lawrence Livermore National Security, LLC. The views and opinions of authors expressed herein do not necessarily state or reflect those of the United States government or Lawrence Livermore National Security, LLC, and shall not be used for advertising or product endorsement purposes.

This work performed under the auspices of the U.S. Department of Energy by Lawrence Livermore National Laboratory under Contract DE-AC52-07NA27344. 


\title{
Thoughts on Uncertainties in the Moments Formalism of the Statistical Theory of Fission Chains
}

\author{
Sean E. Walston* \\ Lawrence Livermore National Laboratory, Livermore, $C A^{\dagger \ddagger}$
}

October 21, 2013

\begin{abstract}
A LANL paper says it provides an uncertainty analysis of subcritical benchmark measurements using the Hage-Cifarelli moment's formalism. The goal of the LANL work is to provide uncertainties in the leakage and total multiplication values for use in subcritical benchmark measurements. Data of the bare BeRP ball taken September 17-20 2012 as part of experiment IER-161 for the Department of Energy Nuclear Criticality Safety Program are used as an example to determine the measured uncertainties in leakage and total multiplication. The contribution of each parameter to the total uncertainty is then examined.
\end{abstract}

\footnotetext{
*Tel.: 925-423-7364. E-mail: walston2@llnl.gov

${ }^{\dagger}$ This document was prepared as an account of work sponsored by an agency of the United States government. Neither the United States government nor Lawrence Livermore National Security, LLC, nor any of their employees makes any warranty, expressed or implied, or assumes any legal liability or responsibility for the accuracy, completeness, or usefulness of any information, apparatus, product, or process disclosed, or represents that its use would not infringe privately owned rights. Reference herein to any specific commercial product, process, or service by trade name, trademark, manufacturer, or otherwise does not necessarily constitute or imply its endorsement, recommendation, or favoring by the United States government or Lawrence Livermore National Security, LLC. The views and opinions of authors expressed herein do not necessarily state or reflect those of the United States government or Lawrence Livermore National Security, LLC, and shall not be used for advertising or product endorsement purposes.

${ }^{\ddagger}$ This work performed under the auspices of the U.S. Department of Energy by Lawrence Livermore National Laboratory under Contract DE-AC52-07NA27344.
} 


\section{INTRODUCTION}

A problematic feature of the LANL paper titled Uncertainty Analysis of Subcritical Benchmark Experiments Using the Hage-Cifarelli Formalism is noted in the section "Uncertainties in the Singles and Doubles Counting Rates" at the bottom of page 1 :

"This work will concentrate on using the singles and doubles rates to solve for neutron multiplication and spontaneous fission rate. The estimation of these rates is performed by fitting the Feynman $Y$ parameter, or $Y_{2}$, as a function of gate-width. Typically chi-squared minimization is the method used for fitting the Feynman $Y$ as a function of amplitude and neutron diffusion lifetime. However, the reduced chi-squared used during this fitting is typically not equal to one. This means the data used for the fit does not follow a normal distribution and hence many of the uncertainty evaluation methods won't work."

Two paragraphs later, in the second paragraph on page 2, one of the above issues is expanded on:

"In the examples presented in this paper, the minimum reduced chisquared values for the fits were on the order of 0.01 or less."

Under fairly general conditions with the method of least-squares in the large sample limit, the $\chi^{2}$ function takes on a Gaussian shape. The importance of the Gaussian distribution stems from the central limit theorem which states that the sum of $n$ independent continuous random variables becomes a Gaussian random variable in the limit of large $n$. The central limit theorem holds regardless of the distribution functions of individual variables. This is the formal justification for treating measurement errors as Gaussian random variables because the total error is nearly always the sum of of a large number of small contributions.

Finding values of the diffusion lifetime $\lambda^{-1}$ and the asymptote $R_{2 \mathrm{~F}}$ that minimize $\chi^{2}$ can be nontrivial because of the nonlinear nature of the fit. One reliable method is to use the Nelder-Mead nonlinear optimization method. The relationship between the true values and confidence regions for $\lambda^{-1}$ and $R_{2 \mathrm{~F}}$ is nonetheless almost certainly not an ellipse as would be the case for a linear two-parameter fit.

Another issue is that the number of degrees of freedom $n_{\mathrm{DOF}}$ is not well defined for any time interval construction where $T$ is chosen in such a way that neutrons used for one value of $T$ get reused for another.

The simplest interpretation for values of $\chi^{2} / n_{\mathrm{DOF}} \lll 1$ (i.e. "reduced chisquared" much less than 1) is that the uncertainties on $Y_{2}$ for a given time interval $T$ are badly overestimated or, more likely, the covariance matrix between different time intervals is incorrect.

Another open question is the size of the quantile $Q_{\gamma}$ that corresponds to a particular confidence interval (e.g. one standard deviation) when the covariance 
matrix between the different time intervals $T$ is not diagonal (i.e. neutrons have been reused across different time gates).

Furthermore, if the "data used for the fit does not follow a normal distribution," then the Neyman construction for confidence intervals should be used. Another issue arises in the construction and interpretation of confidence intervals when a parameter can only take on values in a restricted range. As an important example, consider the case where the mean of a Gaussian distributed variable is constrained on physical grounds to be non-negative. In such cases, one method has been suggested by Feldman and Cousins and applies an ordering principle to the usual Neyman construction.

\section{REVIEW}

The $j$ th combinatorial moment $\mathcal{M}_{j}(T)$ of the multiplicity distribution $b_{n}(T)$ is just the $j$ th factorial moment of $b_{n}(T)$ divided by $j$ !, or more simply

$$
\mathcal{M}_{j}(T)=\sum_{n=j}^{\infty}\left(\begin{array}{c}
n \\
j
\end{array}\right) b_{n}(T)
$$

Furthermore, we define the following constants:

$$
\overline{\nu_{\mu}}=\sum_{\nu=\mu}^{\infty}\left(\begin{array}{l}
\nu \\
\mu
\end{array}\right) C_{\nu}
$$

which are the combinatorial moments of the neutron multiplicity distribution for induced fission, and

$$
\overline{\nu_{\mathrm{S} \mu}}=\sum_{\nu=\mu}^{\infty}\left(\begin{array}{c}
\nu \\
\mu
\end{array}\right) C_{\mathrm{S} \nu}
$$

which are the combinatorial moments of the neutron multiplicity distribution for spontaneous fission.

As a practical matter, the combinatorial moments $\mathcal{M}_{j}(T)$ of the multiplicity distributions $b_{n}(T)$ are easy to compute. The quantities $Y_{j}(T)$ can be expressed in terms of $\mathcal{M}_{j}$ as

$$
\begin{aligned}
Y_{1}(T) & =\mathcal{M}_{1}(T) \\
Y_{2}(T) & =\mathcal{M}_{2}(T)-\frac{\left[\mathcal{M}_{1}(T)\right]^{2}}{2 !} \\
Y_{3}(T) & =\mathcal{M}_{3}(T)-Y_{2}(T) \mathcal{M}_{1}(T)-\frac{\left[\mathcal{M}_{1}(T)\right]^{3}}{3 !} \\
& =\mathcal{M}_{3}(T)-\mathcal{M}_{2}(T) \mathcal{M}_{1}(T)+\frac{\left[\mathcal{M}_{1}(T)\right]^{3}}{3}
\end{aligned}
$$


It is also well known that

$$
\begin{aligned}
& Y_{1}(T)=R_{1} T \\
& Y_{2}(T)=R_{2}\left(T-\frac{1-e^{-\lambda T}}{\lambda}\right) \\
& Y_{3}(T)=R_{3}\left(T-\frac{3-4 e^{-\lambda T}+e^{-2 \lambda T}}{2 \lambda}\right)
\end{aligned}
$$

By summing the contributions from fission chains initiated by both induced and spontaneous fission, it is well known that

$$
R_{j}=\epsilon^{j}\left\{\begin{array}{cc}
F_{\mathrm{I}} M_{\mathrm{e}}+F_{\mathrm{S}} M_{\mathrm{e}} \overline{\nu_{\mathrm{S} 1}} & j=1 \\
F_{\mathrm{I}} M_{\mathrm{e}}^{2} \frac{M_{\mathrm{e}}-1}{\bar{\nu}-1} \overline{\nu_{2}} & j=2 \\
+F_{\mathrm{S}} M_{\mathrm{e}}^{2}\left[\overline{\nu_{\mathrm{S} 2}}+\frac{M_{\mathrm{e}}-1}{\bar{\nu}-1} \overline{\nu_{\mathrm{S} 1}} \overline{\nu_{2}}\right] & \\
F_{\mathrm{I}} M_{\mathrm{e}}^{3} \frac{M_{\mathrm{e}}-1}{\bar{\nu}-1}\left[\overline{\nu_{3}}+2 \frac{M_{\mathrm{e}}-1}{\bar{\nu}-1}{\overline{\nu_{2}}}^{2}\right] & \\
+F_{\mathrm{S}} M_{\mathrm{e}}^{3}\left[\overline{\nu_{\mathrm{S} 3}}+\frac{M_{\mathrm{e}}-1}{\bar{\nu}-1}\left(\overline{\nu_{\mathrm{S} 1}} \overline{\nu_{3}}+2 \overline{\nu_{\mathrm{S} 2}} \overline{\nu_{2}}\right)\right. & \\
\left.+2\left(\frac{M_{\mathrm{e}}-1}{\bar{\nu}-1}\right)^{2} \overline{\nu_{\mathrm{S} 1}}{\overline{\overline{\nu_{2}}}}^{2}\right] & j=3
\end{array}\right.
$$

It is convenient to define the following:

$$
\begin{aligned}
Y_{2 \mathrm{~F}}(T) & =\frac{Y_{2}(T)}{Y_{1}(T)} & (11) & Y_{3 \mathrm{~F}}(T) & =\frac{Y_{3}(T)}{Y_{1}(T)} \\
R_{2 \mathrm{~F}} & =\frac{R_{2}}{R_{1}} & (12) & R_{3 \mathrm{~F}} & =\frac{R_{3}}{R_{1}}
\end{aligned}
$$

Applying Eqs. 7, 8 and 9, the quantities normally used in the analysis are found to be

$$
\begin{aligned}
& Y_{2 \mathrm{~F}}(T)=R_{2 \mathrm{~F}}\left(1-\frac{1-e^{-\lambda T}}{\lambda T}\right) \\
& Y_{3 \mathrm{~F}}(T)=R_{3 \mathrm{~F}}\left(1-\frac{3-4 e^{-\lambda T}+e^{-2 \lambda T}}{2 \lambda T}\right)
\end{aligned}
$$

or, as expressed in terms of the combinatorial moments of the count distributions,

$$
\begin{aligned}
Y_{2 \mathrm{~F}}(T) & =\frac{\mathcal{M}_{2}(T)}{\left[\mathcal{M}_{1}(T)\right]}-\frac{\left[\mathcal{M}_{1}(T)\right]}{2 !} \\
Y_{3 \mathrm{~F}}(T) & =\frac{\mathcal{M}_{3}(T)}{\left[\mathcal{M}_{1}(T)\right]}-\mathcal{M}_{2}(T)+\frac{\left[\mathcal{M}_{1}(T)\right]^{2}}{3}
\end{aligned}
$$


It is worth noting that

$$
\begin{aligned}
\lim _{\lambda T \rightarrow 0} Y_{2 \mathrm{~F}} & =0 \\
\lim _{\lambda T \rightarrow 0} Y_{3 \mathrm{~F}} & =0 \\
\lim _{\lambda T \rightarrow \infty} Y_{2 \mathrm{~F}} & =R_{2 \mathrm{~F}} \\
\lim _{\lambda T \rightarrow \infty} Y_{3 \mathrm{~F}} & =R_{3 \mathrm{~F}}
\end{aligned}
$$

\section{ERROR PROPAGATION}

Let $N$ be the number of time intervals of duration $T$ which are examined, and let $B_{n}(T)$ be the number of those time intervals in which $n$ neutrons were detected. So in other words, suppose that during the first time interval, six neutrons were counted; $B_{6}$ would be incremented by one. During the next time interval, say eight neutrons were counted; $B_{8}$ would be incremented by one, and so on for all $N$ time intervals. In this way, the count distribution $B_{n}(T)$ is built up. The multiplicity distribution $b_{n}(T) \approx B_{n}(T) / N$ is just the probability of counting $n$ neutrons during a time interval of duration $T$, assuming $N \ggg 1$. The total number of neutrons counted $n_{\text {Total }}$ during all $N$ time intervals is

$$
n_{\text {Total }}=\sum_{n=0}^{\infty} n B_{n}
$$

The count distribution $B_{n}(T)$ is fundamentally a multinomial distribution: For a given time interval of duration $T$, there are $N$ independent observations with $B_{0}$ cases of zero neutrons counted, $B_{1}$ cases of one neutron counted, $B_{2}$ cases of two neutrons counted, and so on up to some $B_{n_{\max }}$ cases of $n_{\max }$ neutrons counted. The total number of time intervals $N$ is a fixed number however with

$$
N=\sum_{n=0}^{n_{\max }} B_{n}
$$

The numbers for any two $B_{n}(T)$ are negatively correlated; if there are a greaterthan-average number of time intervals where a particular number $n$ of neutrons were counted, then the probability is increased that there are a fewer-thanaverage number of time intervals where a different number $m(m \neq n)$ of neutrons have been counted. In general, the covariance matrix for a multinomial distribution is

$$
V_{m n}= \begin{cases}-N b_{m} b_{n} & m \neq n \\ N b_{n}\left(1-b_{n}\right) & m=n\end{cases}
$$


where, again, $b_{n} \approx B_{n} / N$. This can easily be written in terms of the count distributions as

$$
V_{m n}= \begin{cases}-\frac{B_{m} B_{n}}{N} & m \neq n \\ B_{n}\left(1-\frac{B_{n}}{N}\right) & m=n\end{cases}
$$

Define the row vectors

$$
\begin{aligned}
& \mathbf{D}_{2 \mathrm{~F}}=\frac{\partial Y_{2 \mathrm{~F}}}{\partial B_{n}} \\
& \mathbf{D}_{3 \mathrm{~F}}=\frac{\partial Y_{3 \mathrm{~F}}}{\partial B_{n}}
\end{aligned}
$$

where it can easily be shown that the elements of the row vectors are

$$
\begin{aligned}
& \frac{\partial Y_{2 \mathrm{~F}}}{\partial B_{n}}=\left(\begin{array}{c}
n \\
2
\end{array}\right) \frac{1}{N \mathcal{M}_{1}(T)}-\frac{n Y_{2 \mathrm{~F}}}{N \mathcal{M}_{1}(T)}-\frac{n}{N} \\
& \frac{\partial Y_{3 \mathrm{~F}}}{\partial B_{n}}=\left(\begin{array}{c}
n \\
3
\end{array}\right) \frac{1}{N \mathcal{M}_{1}(T)}-\left(\begin{array}{c}
n \\
2
\end{array}\right) \frac{1}{N}+\frac{n \mathcal{M}_{1}(T)}{2 N}-\frac{n Y_{2 \mathrm{~F}}}{N}-\frac{n Y_{3 \mathrm{~F}}}{N \mathcal{M}_{1}(T)}
\end{aligned}
$$

The variance on $Y_{2 \mathrm{~F}}$ and $Y_{3 \mathrm{~F}}$ for a given $T$ would then be calculated in the usual way as

$$
\begin{aligned}
\sigma_{Y_{2 \mathrm{~F}}}^{2} & =\mathbf{D}_{2 \mathrm{~F}} V \mathbf{D}_{2 \mathrm{~F}}^{T} \\
\sigma_{Y_{3 \mathrm{~F}}}^{2} & =\mathbf{D}_{\mathbf{3 F}} V \mathbf{D}_{3 \mathrm{~F}}^{T}
\end{aligned}
$$

where the superscript $T$ in these two equations denotes transpose.

Values for $\lambda$ and $R_{2 \mathrm{~F}}$ are determined by minimizing the quantity

$$
\chi^{2}=\mathbf{E}_{\mathbf{2 F}}{ }^{T} W^{-1} \mathbf{E}_{\mathbf{2 F}}
$$

where $W_{i j}$ is the covariance between time gates $T_{i}$ and $T_{j}$ and where the error vector

$$
\mathbf{E}_{\mathbf{2 F}}=\frac{\mathcal{M}_{2}\left(T_{i}\right)}{\mathcal{M}_{1}\left(T_{i}\right)}-\frac{\mathcal{M}_{1}\left(T_{i}\right)}{2 !}-R_{2 \mathrm{~F}}\left(1-\frac{1-e^{-\lambda T_{i}}}{\lambda T_{i}}\right)
$$

is constructed from Eqs. 17 and 15 and is understood to be a column vector corresponding to different values for $T_{i}$.

The quantity $R_{3 \mathrm{~F}}$ and a redundant value for $\lambda$ are similarly determined by minimizing the quantity

$$
\chi^{2}=\mathbf{E}_{3 \mathrm{~F}}{ }^{T} W^{-1} \mathbf{E}_{3 \mathrm{~F}}
$$


where

$$
\mathbf{E}_{3 \mathrm{~F}}=\frac{\mathcal{M}_{3}\left(T_{i}\right)}{\mathcal{M}_{1}\left(T_{i}\right)}-\mathcal{M}_{2}\left(T_{i}\right)+\frac{\left[\mathcal{M}_{1}\left(T_{i}\right)\right]^{2}}{3}-R_{3 \mathrm{~F}}\left(1-\frac{3-4 e^{-\lambda T_{i}}+e^{-2 \lambda T_{i}}}{2 \lambda T_{i}}\right)
$$

The covariance matrix $W$ depends on the structure of the time gates. $W$ depends on how the different values of $T$ are chosen: if the same neutron counts are used to populate count distributions with different values of $T, W$ will not be diagonal. In particular, if any of the neutrons are reused in multiple time gates (different values of $T$ ), then $W$ can become very complex.

Because the $\chi^{2}$-fit can trace its lineage to the method of maximum likelihood,

$$
\chi^{2}\left(\hat{\theta}+\sigma_{\theta}\right)=\chi^{2}(\hat{\theta})+Q_{\gamma}
$$

where $\hat{\theta}=\left(\widehat{R_{2 \mathrm{~F}}}, \widehat{\lambda}\right)$ is the vector of values that minimizes $\chi^{2}$ (e.g. Eq. 33) and $Q_{\gamma}$ is the quantile which defines the confidence region.

$$
\chi_{1 \sigma}^{2}=\mathbf{E}_{\mathbf{2 F}}{ }^{T} W^{-1} \mathbf{E}_{\mathbf{2 F}}-\left[\chi^{2}\left(\widehat{R_{2 \mathrm{~F}}}, \widehat{\lambda}\right)+Q_{\gamma}\right]
$$

to determine $R_{2 \mathrm{~F}}^{\prime}$ and $\lambda^{\prime}$. The statistical errors on $R_{2 \mathrm{~F}}$ and $\lambda$ were then computed as

$$
\begin{aligned}
\sigma_{R_{2 \mathrm{~F}}} & =\left|R_{2 \mathrm{~F}}^{\prime}-\widehat{R_{2 \mathrm{~F}}}\right| \\
\sigma_{\lambda} & =\left|\lambda^{\prime}-\widehat{\lambda}\right|
\end{aligned}
$$

\section{References}

[1] K. Nakamura et al. (Particle Data Group), Review of particle physics, J. Phys., G 37, 075021 (2010).

[2] G. Cowan, Statistical Data Analysis (Oxford University Press Inc., New York, 1998).

[3] Matthews, J. and Walker, R. L. (1970). Mathematical Methods of Physics, Second Edition. Menlo Park: Addison-Wesley Publishing Company, Inc.

[4] Feynman, R. P. (1946). "Statistical Behavior of Neutron Chains," Los Alamos Report LA-591 (classified).

[5] Feynman, R. P., de Hoffmann, F., and Serber, R. (1956). "Dispersion of the Neutron Emission in U-235 Fission," Journal of Nuclear Energy 3, 64.

[6] Bell, G. I. (1963). "Probability Distribution of Neutrons and Precursors in a Multiplying Assembly." Annals Phys. 21, 243. 
[7] Hage, W. and Cifarelli, D. M. (1985). "Correlation Analysis with Neutron Count Distributions in Randomly or Signal Triggered Time Intervals for Assay of Special Nuclear Materials," Nucl. Sci. Eng. 89, 159.

[8] Bohnel, K. (1985). "The Effect of Multiplication on the Quantitative Determination of Spontaneously Fissioning Isotopes by Neutron Correlation Analysis," Nucl. Sci. Eng. 90, 75.

[9] Hage, W. and Cifarelli, D. M. (1985). "On the Factorial Moments of the Neutron Multiplicity Distribution of Fission Cascades," Nucl. Instr. and Meth. A236, 165.

[10] Hage, W. and Cifarelli, D. M. (1986). "Models for a Three-Parameter Analysis of Neutron Signal Correlation Measurements for Fissile Material Assay," Nucl. Instr. and Meth. A251, 550.

[11] Prasad, M. and Snyderman, N. (2002). "Statistical Theory of Fission Chains and Generalized Poisson Neutron Counting Distributions," Lawrence Livermore National Laboratory Report UCRL-ID-148010. To appear in the November 2012 issue of Nuclear Science and Engineering.

[12] Prasad, M. and Snyderman, N. (2005). "Appendix A: Theory of Fission Chains and Count Distributions," Lawrence Livermore National Laboratory Technical Report UCRL-TR-218042. 